\title{
BMJ Open Evaluating the sustainability of differentiated service delivery interventions for stable ART clients in sub-Saharan Africa: a systematic review protocol
}

\author{
Nwanneka Ebelechukwu Okere (D) , ${ }^{1}$ Lisa Urlings, ${ }^{2}$ Denise Naniche, ${ }^{3}$ \\ Tobias F Rinke de Wit, ${ }^{1}$ Gabriela B Gomez, ${ }^{4}$ Sabine Hermans ${ }^{1}$
}

To cite: Okere NE, Urlings L, Naniche D, et al. Evaluating the sustainability of differentiated service delivery interventions for stable ART clients in subSaharan Africa: a systematic review protocol. BMJ Open 2020;10:e033156. doi:10.1136/ bmjopen-2019-033156

- Prepublication history and additional material for this paper are available online. To view these files, please visit the journal online (http://dx.doi. org/10.1136/bmjopen-2019033156).

GBG and SH are joint senior authors.

Received 23 July 2019

Revised 18 December 2019 Accepted 20 December 2019

Check for updates

(c) Author(s) (or their employer(s)) 2020. Re-use permitted under CC BY. Published by BMJ.

For numbered affiliations see end of article.

Correspondence to Ms Nwanneka Ebelechukwu Okere; n.okere@aighd.org

\section{ABSTRACT}

Introduction In 2015, WHO recommended immediate treatment for people living with HIV (PLHIV). As a result, the number of PLHIV needing antiretroviral therapy (ART) in sub-Saharan Africa (SSA) doubled from 12 million to over 25 million. This put a strain on already weak health systems and inspired the exploration of innovative service delivery models—differentiated service delivery (DSD). In DSD, services are tailored according to client clinical type and offer much-needed improvement in efficiency. The potential of achieving good outcomes for both clients and the health system plus the promise of sustainability motivates DSD promotion especially in low-income and middle-income countries. This review aims to evaluate the sustainability of DSD interventions.

Methods and analysis We will systematically review peer-reviewed English literature published between 2000 and 2019 identified by searching PubMed and EMBASE databases. Main inclusion criteria comprise studies describing DSD interventions conducted in SSA focused on stable adult ART clients, whether described alone or compared with clinic-based service delivery. Quality of included studies will be assessed employing the Down and Black's and Joanne Briggs Institute checklists for quantitative and qualitative studies, respectively. We will apply a comprehensive sustainability framework including 40 individual constructs to evaluate, score and rank each intervention for sustainability. Narrative and quantitative synthesis will be conducted as appropriate.

Ethics and dissemination No ethical approval is required for this study as it is a review of published or publicly available data. Review results will be published in a peer-reviewed journal and presented at international conferences.

PROSPERO registration number CRD42019120891.

\section{INTRODUCTION}

In 2015, WHO recommended treatment initiation for people living with HIV (PLHIV), a 'universal test and treat' strategy. As a result, the number of PLHIV needing antiretroviral therapy (ART) in sub-Saharan Africa
Strengths and limitations of this study

- The current systematic review will assess retrospectively the sustainability of differentiated service delivery (DSD) models for HIV treatment in terms of their impact.

- We aim to equip policy-makers with information necessary for making rational choices to prevent wastage of limited resources by providing an evaluation of the sustainability of DSD interventions.

- The comparability of results could be a challenge because a wide range of interventions have been reported and consequently a wide range of outcomes are expected to be included.

- We will review interventions conducted in subSaharan Africa and articles published in English leading to a possible bias by excluding articles in other languages.

(SSA) more than doubled from 12 million to over 25 million. ${ }^{12}$ This increase in demand adds strain on already weak health systems. It made obvious the insufficiency of traditional clinic-based care and called for innovative service delivery models. Differentiated service delivery (DSD) prioritises client preferences while also aiming to increase efficiency. ${ }^{3-8}$ DSD has evolved over time and encompasses a wide range of concepts in programme implementation especially in low-income and middle-income countries such as task shifting, decentralisation and community-based ART. Ultimately, the goal of DSD is to increase access to and retention in care by reducing demand-side and supplyside barriers of clinic-based models of service delivery, especially for rural populations. ${ }^{9-12}$

Differentiated care became necessary as more clients were initiated on lifesaving antiretrovirals and ART clinics were becoming 
crowded. This largely influenced the public health approach involving decentralisation recommended by WHO as far back as 2006. ${ }^{13}{ }^{14}$ Since then, attempts have been made to streamline services by categorising clients in terms of their clinical profile, such as being 'stable'. Currently, WHO defines a stable client as: 'being on ART for at least 1 year, no current illnesses or pregnancy, a good understanding of lifelong adherence and evidence of treatment success (two consecutive viral load measurements below 1000 copies $/ \mathrm{mL}$ )'. ${ }^{1}$

Four DSD models were recently described which focus on stable ART clients. ${ }^{15} 16$ They include (1) healthcare worker (HCW) groups, (2) facility-based individual models, (3) client managed groups and (4) out-of-facility individual models. Each model is defined by four dimensions, namely 'who' provides care, 'what' care is provided, 'when' do the activities happen and 'where' is care provided. Prepackaged ART refill, symptom screening, health talks, clinical consultations or referral if unwell defines 'what' care is provided across models. 'When' care is provided, as a major benefit of DSD, is usually less frequent than the routine monthly visits for clients categorised as stable. Though WHO recommends 3-6 months intervals, anything between 2 and 12 months is possible depending on the context. But these models are characterised by the other two dimensions, that is, 'who' provides care (the cadre of healthcare personnel involved) and 'where' care is provided (location). Interventions may be conducted by HCWs within facilities, for example, adherence clubs (ACs), fast-track ART refill, 6-monthly appointments, multimonth scripting or by community-based HCWs, for example, community drug distribution points. Interventions can also be conducted by non-HCWs within facilities, for example, ACs or by community-based non-HCWs, for example, community ART refill groups, community ART groups, mobile outreach (OR) and AC (see table 1$){ }^{17}$

The potential for achieving a lasting impact and sustainability is the push behind DSD promotion. Sustainability in healthcare is a broad concept which was only recently defined as "programme, clinical intervention and/or implementation strategies which continue to be delivered after a defined period of time (ie, after expiration of initial external funding) including the maintenance of programme or individual behavioural change (ie, clinician, client) which may evolve or adapt while continuing to produce benefits for individuals/systems' ${ }^{18}$ Several conceptual frameworks have been described articulating the constructs mentioned in this definition. ${ }^{19-25}$ These frameworks consolidate and elaborate on sustainability as an important concept embedded within the continuum of implementation science. ${ }^{26-28}$ Most of them view sustainability as a process to be prospectively explored during implementation rather than an outcome to be retrospectively evaluated. Lennox et $a l,{ }^{29}$ in a recent review, developed a comprehensive framework of 40 individual sustainability constructs categorised into six broad domains, namely (1) intervention design and delivery, (2) intervention processes, (3) external environment, (4) organisational setting, (5) resources and (6) people involved. The Lennox framework is the basis used in this review.

\section{Why is it important to do this review?}

The long-term sustainability of HIV programme interventions is essential as lifelong ART remains a necessity for PLHIV. Therefore, sustainability underlies strategies endorsed by HIV programme, for example, task shifting, decentralisation of care, differentiation of care.$^{1530-35}$ The increasing international call for universal health coverage is similarly motivated (in addition to other considerations such as equity) and will likely benefit not just HIV programme but entire health systems. ${ }^{36}{ }^{37}$ While many DSD interventions have been implemented within HIV programme with good outcomes, knowledge about their sustainability has been limited. ${ }^{38-40}$ Furthermore, it is not clear how these interventions can be properly integrated within the existing health system to ensure they last.

Research on the sustainability of health interventions has been hampered by a lack of consensus. For example, several definitions have existed, some referring to the continuation of an intervention as a whole or in part ${ }^{41}$

Table 1 Characterisation of DSD models

Location (where)

Within facilities

$\begin{array}{lll}\begin{array}{l}\text { Personnel } \\ \text { (who) }\end{array} & \text { HCW managed } & \begin{array}{l}\text { Fast-track ART refill } \\ \text { Six-monthly appointment } \\ \text { Multimonth scripting }\end{array} \\ & & \begin{array}{l}\text { Adherence clubs (ACs) } \\ \text { (model 1) }\end{array} \\ & & \text { ACs } \\ & \text { (model 3) } \\ & \end{array}$

Community based

ACs

Community drug distribution points (model 2)

Community ART groups Community ART refill groups ACs Mobile outreach (OR) (model 4) 
and others to the continuation of benefits of an intervention. ${ }^{42}$ Similarly, several sustainability frameworks exist, making comparability across studies difficult. Common grounds are, however, beginning to emerge. The framework by Shediac-Rizkallah and Bone ${ }^{43}$ provided the basic constructs on which other sustainability studies continue to build. ${ }^{4-46}$ The recent syntheses of a comprehensive definition and framework, both anchored in this framework, provided useful tools which we have adopted to gain insight into the sustainability of DSD models. ${ }^{18} 29$

In resource-constrained settings, the promising clientrelated outcomes and the reduced demand on resources it places on health systems without undermining effectiveness are thought to make DSD feasible to implement. HIV programme will, therefore, require evidence to inform which DSD options to explore. Our review aims to fill this gap by evaluating the sustainability of available DSD interventions.

While DSD interventions may not be as onerous as standard clinical care, they pose some demands to the health system. As countries in SSA strive to increase their domestic share of funding for their HIV programmes, they must be aware of the aspects influencing sustainability of the DSD options considered. This review will provide information necessary to inform these discussions. Our review also aims to provide the impetus for further enquiry into service delivery models and socioeconomic issues necessary to ensure continual client empowerment while supporting minimally resourced health systems.

\section{METHODS AND ANALYSIS}

The protocol adheres to the Preferred Reporting Items for Systematic Reviews and Meta-Analyses Protocols guidelines. $^{47}$

\section{Search strategy}

We will search PubMed and EMBASE to identify eligible articles using a comprehensive search strategy (see online supplementary file I). Additionally, the reference lists of included articles and the reference lists of previously published reviews will be reviewed for other relevant articles. The review period of interest will be between 2000 and 2019 to capture all articles published since the widespread scale-up of ART services across SSA. Only English language articles will be included. We will use Rayyan Qatar Computing Research Institute (Rayyan QCRI) app, the free systematic reviews web application ${ }^{48}$ to store, organise and manage eligible references whose titles and abstracts will be screened. A follow-up search will be conducted prior to the completion of the review which is planned 9 months after commencement.

\section{Eligibility criteria}

The inclusion and exclusion criteria of articles are as listed- see box 1. Appropriate definitions to guide the selection of articles are also provided-see box 2 .
Box 1 Eligibility criteria

\section{Inclusions}

- Observational, experimental or quasi-experimental studies.

- Studies involving stable adult antiretroviral therapy clients accessing HIV care in sub-Saharan Africa.

- Studies describing or assessing HIV services delivered through models other than standard clinic-based care.

- Studies which compare the performance of these other service delivery models with standard clinic-based HIV service delivery accessed by other clients. Though, lack of this comparison is not an exclusion criterion.

\section{Exclusions}

- Reviews, editorials, protocol studies and clinical guidelines.

- Studies describing or assessing interventions focussed on special population groups for example, adolescents, children, pregnant women, men who have sex with men, commercial sex workers.

- Studies utilising data retrospectively collected in electronic databases with little description of the actual intervention.

\section{Selection of studies}

Two reviewers (NEO and LU) will independently screen the titles and abstracts of articles identified in the searches to identify potential articles. The reviewers will then conduct full-text reading of these potential articles to determine eligibility. GBG and $\mathrm{SH}$ will be engaged in discussions to make a final determination on eligibility whenever the two reviewers are unable to reach a consensus. Reasons for exclusions will be reported.

\section{Data extraction}

We have developed a data extraction form. NEO and LU will pilot the data extraction form on a subset of five articles to assess its functionality. After incorporating feedback obtained from the review team (NEO, LU, GBG and $\mathrm{SH}$ ), a final form will then be used to extract relevant data independently by the two reviewers.

Data to be extracted will include: (1) details of each intervention including site, setting, service delivery model, funding, providers, period, theoretical underpinning; (2) study population, participant type and numbers; (3) sustainability constructs as described in table 2 and (4) challenges or unintended consequences faced during implementation, comments, clients and staff perspectives (see online supplementary file II).

\section{Box 2 Definitions}

\section{Stable antiretroviral therapy (ART) clients}

Definition of a stable ART client has been fine-tuned over time, therefore, there will be no restriction in terms of definition. We will rely on the studies' definitions and will provide a summary of definitions employed.

\section{Adult clients}

The definition of adult clients varies across studies. We will define adult as $\geq 18$ years but will include studies if the age range of participants is not $<15$ years. 
Table 2 Sustainability constructs and evidence to be extracted (adapted from Lennox et $\mathrm{al}^{29}$ ) (primary study outcomes)

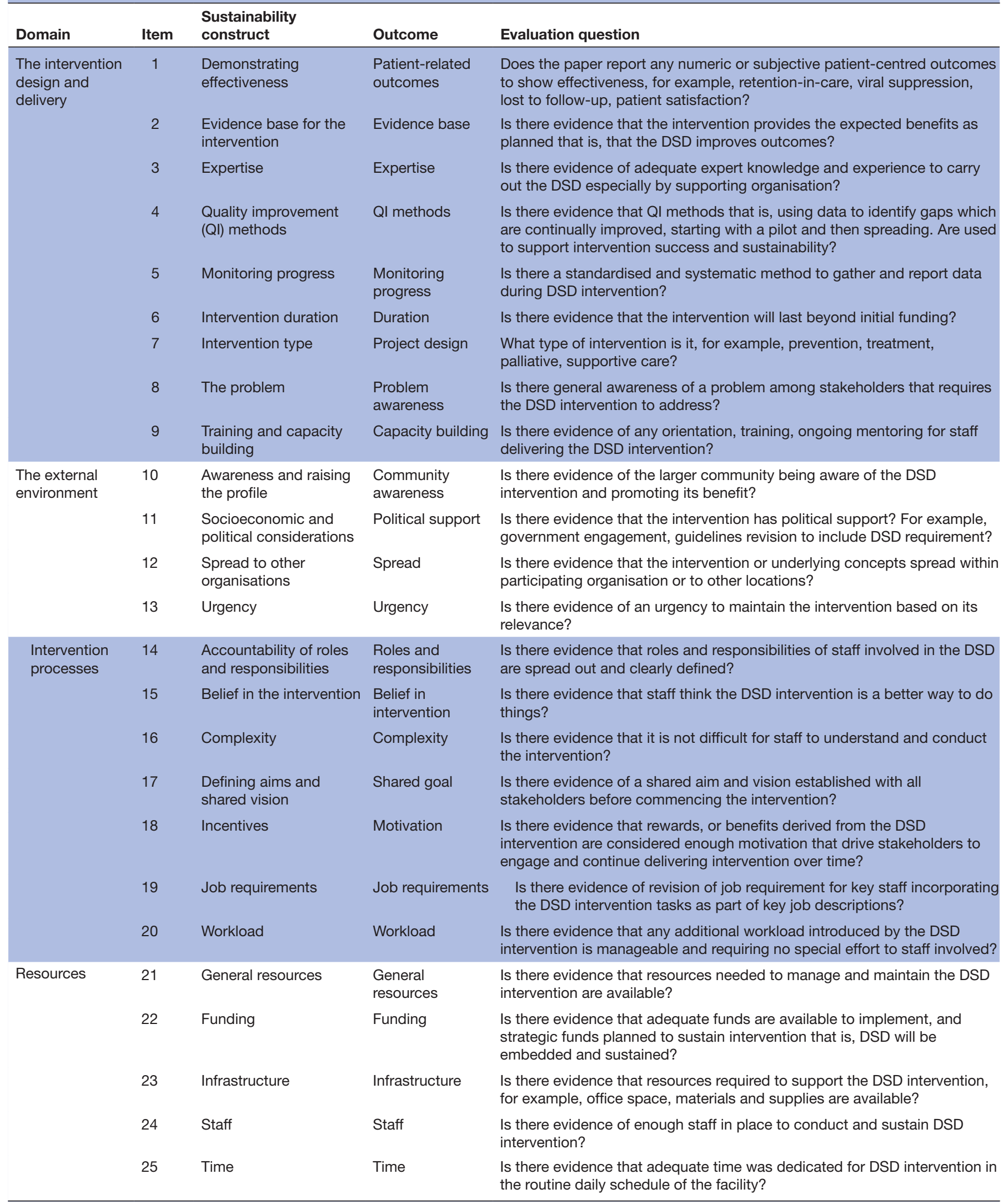




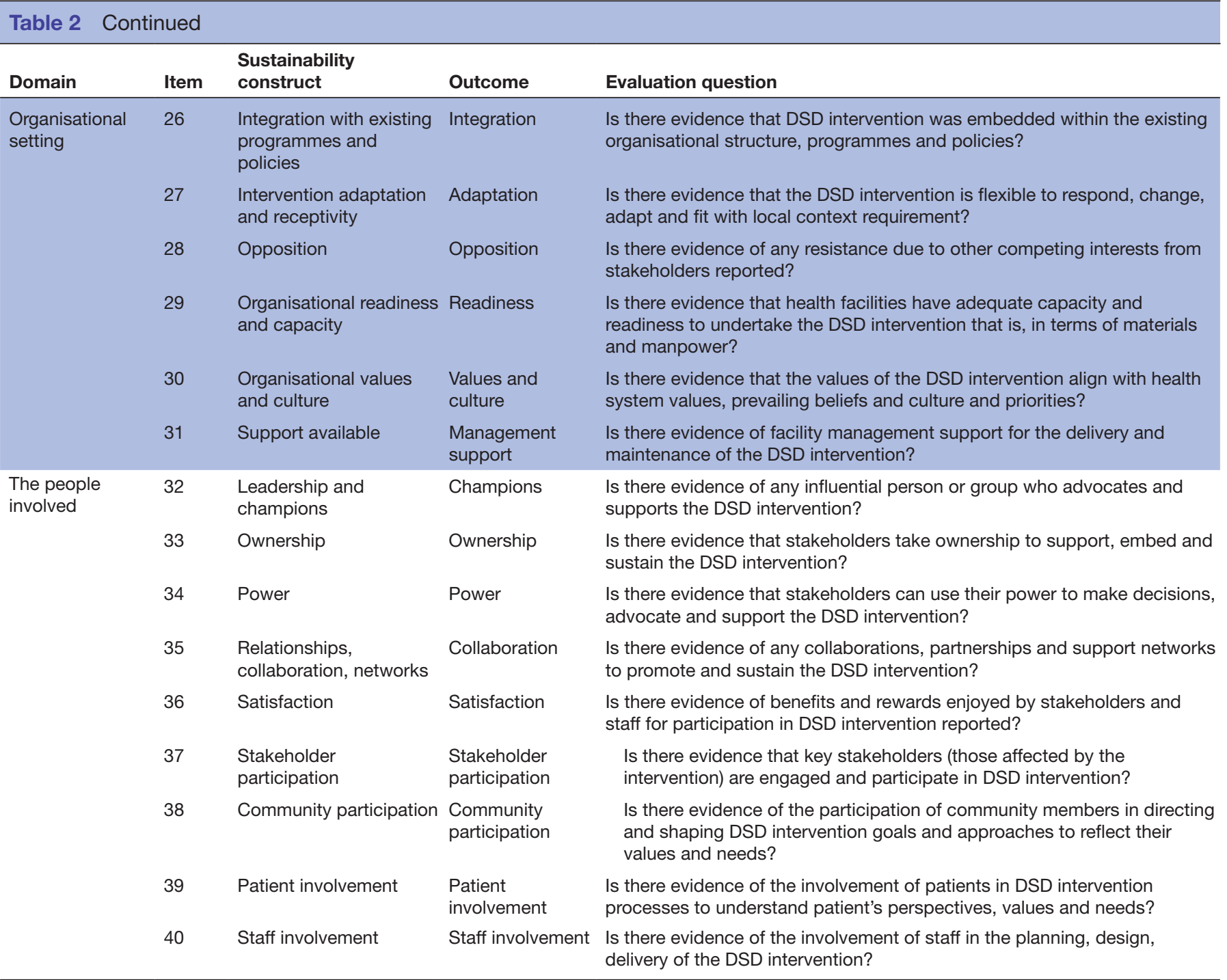

DSD, differentiated service delivery.

We speculate that information regarding some constructs in the framework may be under-reported in the included articles. However, by describing the extent to which this under-reporting is happening, we hope to highlight this issue for further research. We will quantify and report the levels of missing information observed during data extraction (estimated by the number of 'not described' (ND) assigned by reviewers) per construct for included studies.

\section{Data analysis}

Primary outcome measures will include the scores and rankings on sustainability constructs and domains as defined by Lennox et $a l^{29}$ and presented in table 2 . Since the review aims to extract and appraise evidence of sustainability constructs retrospectively, we simplified a previously described tool to assess sustainability in ongoing projects. ${ }^{49}$ The two reviewers will assign a score of 3, 2 or 1 independently based on their assessment of whether there was enough, some or no evidence about each construct (see online supplementary file II).
Additionally, "ND" will be assigned if the reviewers are not able to make an assessment with information provided. Attempts will be made to contact study authors directly for any additional data considered necessary. Secondary outcome measures will include (1) trends observed in sustainability scores as a result of variations in the criteria employed to define stable clients, (2) if appropriate, quantitative intervention outcome measures such as retention in care, viral suppression, lost to follow-up, client or provider costs reported across similar interventions and (3) qualitative outcomes such as perspectives of clients on issues such as satisfaction with intervention or HCW, access to ART, peer support and perspectives of HCWs on workload or care for sick clients among others. Qualitative outcomes will be extracted as text.

Qualitative and quantitative analyses will be conducted. The basic characteristics, key findings including strengths and challenges of DSD interventions will be summarised. For qualitative analysis, we will conduct narrative synthesis ${ }^{50}$ to identify commonly occurred themes. For 
quantitative analysis, we will employ descriptive statistics to determine the sustainability performance of DSD interventions. Performance will be measured in terms of overall construct scores and percentages and median domain scores. This will then be used to rank constructs, domains, interventions and models for sustainability with higher scores ranked as better sustainability.

\section{Quality of evidence}

Two reviewers, NEO and LU, will assess risk of bias in included studies using the Downs and Black checklist. ${ }^{51}$ The following domains will be assessed: quality of reporting, external validity and internal validity, confounding (selection bias) and power. For qualitative studies, we will assess risk of bias using the Joanna Briggs Institute checklist. ${ }^{52}$ Disagreements between reviewers will be resolved as for study selection. The overall quality of included studies will be evaluated, if possible, using the Grading of Recommendations Assessment, Development and Evaluation framework for quality of evidence. ${ }^{53}$

\section{ETHICS AND DISSEMINATION}

Results and findings of the review will be published in a peer-reviewed journal and presented at appropriate conferences and meetings.

\section{Author affiliations}

${ }^{1}$ Global Health, University of Amsterdam, Amsterdam Institute for Global Health and Development, Amsterdam, The Netherlands

${ }^{2}$ Medicine, Amsterdam University Medical Centres, Amsterdam, Noord-Holland, The Netherlands

${ }^{3}$ Global Health, University of Barcelona, Barcelona Institute for Global Health, Barcelona, Catalunya, Spain

${ }^{4}$ Global Health and Development, London School of Hygiene and Tropical Medicine, London, UK

\section{Twitter Nwanneka Ebelechukwu Okere @onetweet10}

Contributors NEO, GBG and SH conceived the review design and in refining and registering the review protocol. NEO, GBG, SH and LU contributed to the search strategy. NEO drafted the initial manuscript. NEO, GBG, SH, DN and TFRdW provided content expertise. All authors contributed to revisions to obtain the final manuscript. All authors read and approved the final manuscript.

Funding This work was supported by Erasmus Mundus Joint Doctorate Fellowship, Framework Partnership Agreement 2013-0039, Specific Grant Agreement 2014-0681.

\section{Competing interests None declared.}

Patient and public involvement statement We will be reviewing and assessing already conducted interventions retrospectively and this will permit little or no involvement of patients and the public.

Patient consent for publication Not required.

Ethics approval No ethical approval is required for the conduct of this systematic review since all data used are available in the public space.

Provenance and peer review Not commissioned; externally peer reviewed.

Open access This is an open access article distributed in accordance with the Creative Commons Attribution 4.0 Unported (CC BY 4.0) license, which permits others to copy, redistribute, remix, transform and build upon this work for any purpose, provided the original work is properly cited, a link to the licence is given, and indication of whether changes were made. See: https://creativecommons.org/ licenses/by/4.0/.

ORCID iD
Nwanneka Ebelechukwu Okere http://orcid.org/0000-0001-9182-6518

\section{REFERENCES}

1 World Health Organization. Consolidated guidelines on the use of antiretroviral drugs for treating and preventing HIV infection: recommendations for a public health approach, 2016. Available: https://www.who.int/hiv/pub/arv/arv-2016/en/

2 Pustil R. Global AIDS update. Aids 2016;17:S3-11.

3 Bango F, Ashmore J, Wilkinson L, et al. Adherence clubs for longterm provision of antiretroviral therapy: cost-effectiveness and access analysis from Khayelitsha, South Africa. Trop Med Int Heal 2016;21:1115-23.

4 Long L, Brennan A, Fox MP, et al. Treatment outcomes and cost-effectiveness of shifting management of stable art patients to nurses in South Africa: an observational cohort. PLoS Med 2011;8:e1001055.

5 Babigumira JB, Castelnuovo B, Stergachis A, et al. Cost effectiveness of a Pharmacy-only refill program in a large urban HIV/ AIDS clinic in Uganda. PLoS One 2011;6:e18193.

6 Grimsrud A, Bygrave H, Doherty M, et al. Reimagining HIV service delivery: the role of differentiated care from prevention to suppression. J Int AIDS Soc 2016;19:21484.

7 Okoboi S, Ding E, Persuad S, et al. Community-Based art distribution system can effectively facilitate long-term program retention and low-rates of death and virologic failure in rural Uganda. AIDS Res Ther 2015;12.

8 Tsondai PR, Wilkinson LS, Grimsrud A, et al. High rates of retention and viral suppression in the scale-up of antiretroviral therapy adherence clubs in Cape town, South Africa. J Int AIDS Soc 2017;20:21649.

9 Bedelu M, Ford N, Hilderbrand K, et al. Implementing antiretroviral therapy in rural communities: the Lusikisiki model of decentralized HIV/AIDS care. J Infect Dis 2007;196:S464-8.

10 Fredlund VG, Nash J. How far should they walk? increasing antiretroviral therapy access in a rural community in northern KwaZulu-Natal, South Africa. J Infect Dis 2007;196:S469-73.

11 Mutevedzi PC, Lessells RJ, Heller T, et al. Scale-Up of a decentralized HIV treatment programme in rural KwaZulu-Natal, South Africa: does rapid expansion affect patient outcomes? Bull World Health Organ 2010;88:593-600.

12 Davis N, Kanagat N, Sharer M, et al. Review of differentiated approaches to antiretroviral therapy distribution. AIDS Care 2018;30:1010-6.

13 WHO Guidelines. Antiretroviral therapy for HIV infection in adults and adolescents: recommendations for a public health approach HIVI AIDS programme important: addendum to 2006 WHO guidelines on antiretroviral therapy for HIV infection in adults and adolescents. WHO, 2006.

14 Gilks CF, Crowley S, Ekpini R, et al. The who public-health approach to antiretroviral treatment against HIV in resource-limited settings. The Lancet 2006;368:505-10.

15 IAS. Differentiated care for HIV: a decision framework for antiretroviral therapy delivery $2016: 1-56$.

16 WHO. Key considerations for differentiated antiretroviral therapy delivery for specific populations: children, adolescents, pregnant and breastfeeding women and key populations 2017

17 International AIDS Society. Differentiated service delivery, 2019. Available: http://www.differentiatedcare.org/ [Accessed 3 Jun 2019].

18 Moore JE, Mascarenhas A, Bain J, et al. Developing a comprehensive definition of sustainability. Implement Sci 2017;12:110.

19 Whelan J, Love P, Pettman T, et al. Cochrane update: predicting sustainability of intervention effects in public health evidence: identifying key elements to provide guidance. J Public Health 2014;36:347-51.

20 Schell SF, Luke DA, Schooley MW, et al. Public health program capacity for sustainability: a new framework. Implementation Sci 2013;8:1.

21 Gruen RL, Elliott JH, Nolan ML, et al. Sustainability science: an integrated approach for health-programme planning. The Lancet 2008;372:1579-89.

22 Hodge LM, Turner KMT. Sustained implementation of evidencebased programs in disadvantaged communities: a conceptual framework of supporting factors. Am J Community Psychol 2016;58:192-210.

23 Mancini JA, Marek LI. Sustaining community-based programs for families: conceptualization and Measurement*. Fam Relat 2004;53:339-47.

24 Sarriot EG, Winch PJ, Ryan LJ, et al. A methodological approach and framework for sustainability assessment in NGO-implemented 
primary health care programs. Int $\mathrm{J}$ Health Plann Manage 2004;19:23-41.

25 Luke DA, Calhoun A, Robichaux CB, et al. The program sustainability assessment tool: a new instrument for public health programs. Prev Chronic Dis 2014;11:130184.

26 Moullin JC, Sabater-Hernández D, Fernandez-Llimos F, et al. A systematic review of implementation frameworks of innovations in healthcare and resulting generic implementation framework. Heal Res Policy Syst 2015;13.

27 Feldstein AC, Glasgow RE. A practical, robust implementation and sustainability model (PriSM) for integrating research findings into practice. Jt Comm J Qual Patient Saf 2008;34:228-43.

28 Theobald S, Brandes N, Gyapong M, et al. Implementation research: new imperatives and opportunities in global health. Lancet 2018;392:2214-28.

29 Lennox L, Maher L, Reed J. Navigating the sustainability landscape: a systematic review of sustainability approaches in healthcare. Implement. Sci 2018;13:27.

30 Callaghan M, Ford N, Schneider H. A systematic review of taskshifting for HIV treatment and care in Africa. Hum Resour Health 2010;8:8.

31 Philips M, Zachariah R, Venis S. Task shifting for antiretroviral treatment delivery in sub-Saharan Africa: not a panacea. Lancet 2008;371:682-4.

32 Kredo T, McCaul M, Volmink J. Task-shifting from doctors to nondoctors for initiation and maintenance of antiretroviral therapy. S Afr Med J 2015;105:626-7.

33 Pasipamire L, Nesbitt RC, Ndlovu S, et al. Retention on art and predictors of disengagement from care in several alternative community-centred art refill models in rural Swaziland. J Int AIDS Soc 2018;21:e25183.

34 Adjetey V, Obiri-Yeboah D, Dornoo B. Differentiated service delivery: a qualitative study of people living with HIV and accessing care in a tertiary facility in Ghana. BMC Health Serv Res 2019;19:95.

35 Prust ML, Banda CK, Nyirenda R, et al. Multi-month prescriptions, fast-track refills, and community art groups: results from a process evaluation in Malawi on using differentiated models of care to achieve national HIV treatment goals. J Int AIDS Soc 2017;20:21650.

36 World Health Organisation (WHO). HIV, universal health coverage and the post-2015 development agenda, 2015.

37 The Global Fund. Building resilient and sustainable systems for health, 2018.

38 Castelli F, Pietra V, Diallo I, et al. Antiretroviral (ARV) therapy in resource poor countries: what do we need in real life? Open AIDS J 2010;4:28-32.
39 Azeredo TB, Oliveira MA, Santos-Pinto CDB, et al. Sustainability of ARV provision in developing countries: challenging a framework based on program history. Cien Saude Colet 2017;22:2581-94.

40 Abebe B. Sustainability of HIV/AIDS care support programmes Acknowledgement-Igratefully acknowledge Prof. Yemane Berhane, program and theory review course. Type Double Blind Peer Rev Int Res J Publ Glob Journals Inc 2012;12.

41 Ament SMC, de Groot JJA, Maessen JMC, et al. Sustainability of professionals' adherence to clinical practice guidelines in medical care: a systematic review. BMJ Open 2015;5:e008073.

42 Wiltsey Stirman S, Kimberly J, Cook N, et al. The sustainability of new programs and innovations: a review of the empirical literature and recommendations for future research. Implement Sci 2012;7:17.

43 Shediac-Rizkallah MC, Bone LR. Planning for the sustainability of community-based health programs: conceptual frameworks and future directions for research, practice and policy. Health Educ Res 1998;13:87-108.

44 Chambers DA, Glasgow RE, Stange KC. The dynamic sustainability framework: addressing the paradox of sustainment amid ongoing change. Implement Sci 2013;8:1.

45 Scheirer MA. Is sustainability possible? A review and commentary on empirical studies of program sustainability. Am J Eval 2005;26:320-47.

46 Iwelunmor J, Blackstone S, Veira D, et al. Toward the sustainability of health interventions implemented in sub-Saharan Africa: a systematic review and conceptual framework. Implement Sci 2016;11:43.

47 Moher D, Shamseer L, Clarke M, et al. Preferred reporting items for systematic review and meta-analysis protocols (PRISMA-P) 2015 statement. Syst Rev 2015;4.

48 Ouzzani M, Hammady H, Fedorowicz Z, et al. Rayyan-a web and mobile APP for systematic reviews. Syst Rev 2016;5:1-10.

49 Calhoun A, Mainor A, Moreland-Russell S, et al. Using the program sustainability assessment tool to assess and plan for sustainability. Prev Chronic Dis 2014:11:130185.

50 Popay J, Roberts H, Sowden A, et al. Guidance on the Conduct of Narrative Synthesis in Systematic Reviews. A Product from the ESRC Methods Programme2006:211-9.

51 Downs SH, Black N. The feasibility of creating a checklist for the assessment of the methodological quality both of randomised and non-randomised studies of health care interventions. J Epidemiol Community Heal 1998;52:377-84

52 Lockwood C, Munn Z, Porritt K. Qualitative research synthesis. Int J Evid Based Healthc 2015;13:179-87.

53 Guyatt G, Oxman AD, Akl EA, et al. Grade guidelines: 1. Introduction-GRADE evidence profiles and summary of findings tables. J Clin Epidemiol 2011;64:383-94. 